http://dx.doi.org/10.12795/PH.1993.v08.i01.19

\title{
ALGUNAS CUESTIONES SOBRE LA OBRA DE BAQUÍLIDES
}

\author{
Antonio Villarrubia
}

1. El propósito de este trabajo es ofrecer algunas notas sobre distintas cuestiones de la poesía de Baquílides, cuyas peculiaridades de contenido y estilo ponen de manifiesto la complejidad de estas composiciones ${ }^{1}$.

2. La oda 1, dedicada a Argeo de Ceos, vencedor en el pugilato infantil en los Juegos Ístmicos -al igual que la oda 2-, una de las de mayor extensión y originalidad del conjunto de su producción, presenta una sección mítica (vv. 19-140a) que aborda una leyenda local de dicha isla, la fundación de la ciudad de Coresia (o Coreso), patria del célebre Euxancio ${ }^{2}$. Estos versos se encuentran en un estado de conservación fragmentario, cuyas líneas maestras, al menos, pueden apreciarse y cuya estructura sería la siguiente: 1 . las hijas de Damón (vv. 19-83), 2. Minos y Dexítea (vv. 111-124) y 3. la descendencia de Minos y Dexítea: Euxancio (vv. 125-140a). El mito muestra, pues, un rico entramadó de temas y motivos literarios entre los que, como primer elemento, destaca una theoxenía: la visita de Zeus y, quizás, Posidón o Apolo a la isla y la acogida hospitalaria de las hijas de Damón, príncipe de los Telquines, en contraste con la actitud hostil de éstos.

Pero sale al paso la cuestión, continuamente soslayada, de la presencia en este poema de los Telquines, parte de aquel pueblo de la isla de Rodo (o Rodas), fulminado por Zeus, que se había asentado en Ceos. Si se hubiera optado por la ausencia, se habría seguido así la tónica de supresión del mundo, lleno de violencia, anterior al establecimiento de las nuevas sociedades culturales en consonancia con el método utilizado por Píndaro. Lo importante es el momento en el que comienza a verse la renovación de un pueblo antiguo, hecho que en este caso sucede al tercer día después de la destrucción de la isla, primero de la estancia de Minos y los demás cretenses (vv. $112 b-115$ y ss.) ${ }^{3}$ :

\footnotetext{
${ }^{1}$ Para una visión de la obra de Baquílides, cf. R. C. Jebb, Bacchylides. The Poems and Fragments (Hildesheim 1967 [Cambridge 1905]), G. W. Pieper, Unity and Poetic Technique in the Odes of Bacchylides (Urbana [Illinois] 1969) y, sobre todo, A. P. Burnett, The Art of Bacchylides (London-Cambridge [Massachusetts] 1985).

${ }^{2}$ Cf., e.g., R. C. Jebb, op. cit. 185-189, 242-251 y 435-451, B. Snell-H. Maehler, Bacchylidis carmina cum fragmentis (Leipzig $1970^{10}$ ) XXXIX-XLI y 1-7 y.H. Maehler, Die Lieder des Bakchylides. Erster Teil. Die Siegeslieder. I. Edition des Textes mit Einleitung und Übersetzung (Leiden 1982) 48-57, II. Kommentar (Leiden 1982) $1-28$.
}

${ }^{3}$ Cf. R. C. Jebb, op. cit. 242, H. Maehler, op. cit. (II) 15 y A. P. Burnett, op. cit: 150-153. 
...Tpเтátal $\mu \in \tau\left[{ }^{\smile--}\right.$

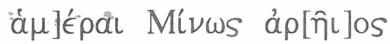

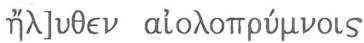

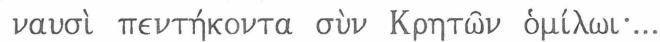

Y todo ello sin dejar de advertir que se trata de una oda que resalta fundamentalmente los aspectos positivos, capaces de iluminar la figura del joven atleta.

Surge, no obstante, el problema de qué hacer con la noticia de la mención de los Telquines en la poesía de Baquílides, ofrecida por J. Tzetzes, que hace a los cuatro Telquines originarios (Acteo, Megalesio, Órmeno y Lico, hermanos de las Erinies) hijos de Tártaro y Némesis frente a quienes los consideraban hijos de Ponto y Gea ${ }^{4}$.

Una postura coherente, a nuestro juicio, es aceptar que los Telquines aparecen en la obra de Baquílides y, probablemente, en esta oda -quizás en los numerosos versos perdidos-, de igual manera que se incluye a los Gigantes, hijos de Gea, en la oda 15 (vv. 62-63), dejando claro, por un lado, que no puede generalizarse la técnica antes aludida de Píndaro, que, además, se sirve del mundo anterior a la paz olímpica definitiva propiciada por Zeus cuando se refiere, por ejemplo, a los Titanes, hijos de Urano y Gea, a los Gigantes, hijos también de Urano y Gea, o a Tifón, hijo de Tártaro y Gea, ${ }^{5}$ y teniendo en cuenta, por otro lado, que los mencionará más tarde Calímaco en varios pasajes ${ }^{6}$.

A pesar del estado de la oda, en la que no se lee el nombre de dicho pueblo, se advierten

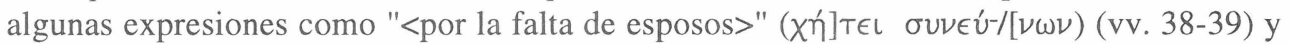
como las palabras de Mácelo, que se lamenta "por una desdicha de doble filo" (ả $\mu] \phi a ́ k \in \mathrm{l}$ Súal) (v. 79) -posiblemente, la destrucción de los suyos y el abandono de los maridos- y un

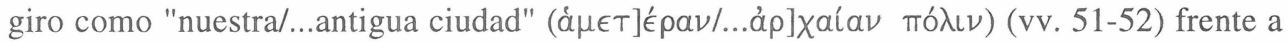

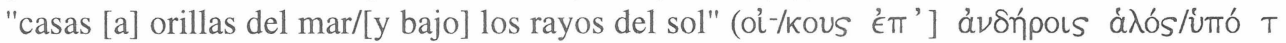

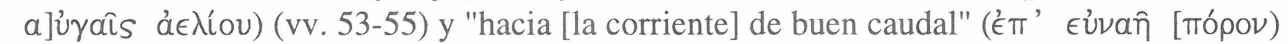
(v. 75), que parecen referirse a él y que llevó incluso a la discutida recuperación del nombre de Damón, cuyas "hijas huyeron/a una ciudad impregnada de sol" ( $<\Delta a ́ \mu \omega \nu o s>a ̊ \lambda] v \xi a \nu$

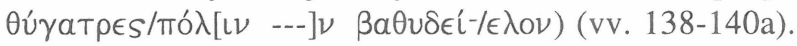

3. La oda 3, dedicada a Hierón de Siracusa, vencedor en la carrera de cuadrigas en los Juegos Olímpicos, por lo demás ampliamente estudiada, presenta un interesante juego de palabras ${ }^{7}$. La función de este recurso es, en nuestra opinión, reforzar aún más la cohesión semántica de la que hace gala, en el mismo plano de los ecos y las recurrencias verbales. En

\footnotetext{
${ }^{4}$ Así en Theogonia 80-86, apud P. Matranga, Anecdota Graeca 580.

${ }^{5}$ En $P .4 .291$ a e Himno 1 a Zeus (=fr. 35 Snell-Maehler), en $P .8 .15-20$ (esp. 17), N. 1. 67-69a y N. 7.90 y en $O$. 4.6-10 (esp. 6a-7), P. 1.14-28, P. 8.15-20 (esp. 16) y frs. 91, 92, 93 y 94 Snell-Maehler), respectivamente.

${ }^{6}$ En Del. (4).28-33, esp. 31, Aet.1.1 Pfeiffer y, sobre todo, Aet. 3.75.64-69 Pfeiffer, una variante mítica del mismo tema baquilideo.

${ }^{7}$ Cf., e.g., R. C. Jebb, op. cit. 189-197, 252-267 y 452-463, B. Snell-H. Maehler, op. cit. XLI y 8-13 y H. Maehler, op. cit. (I) 60-67 y (II) 32-63.
} 
aunque es, sin duda, el que presenta la mayor complejidad y requiere una mayor atención del oyente. Veamos sólo algunos casos de interés: Oda 1: La ciudad de Coresia o Coreso

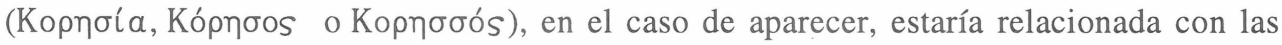

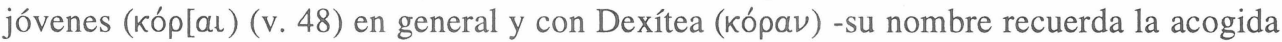
divina- (v. 117) en particular. Oda 5: Deyanira ( $\Delta a \ddot{a} a ́ v \in \iota \rho a \nu)$ (v. 173) recoge el adjetivo

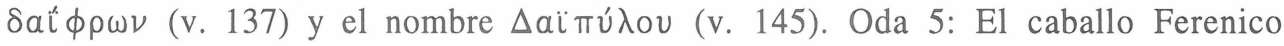

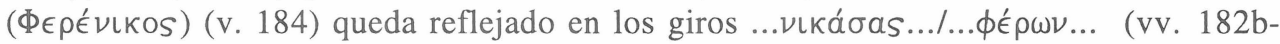
186). Oda 6: El destinatario, Lacón ( $\Lambda \alpha ́ \chi \omega \nu)$ (v. 1), juega con un verbo, $\lambda a ́ \chi \epsilon \in$ (v. 2), que

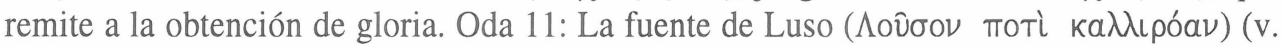

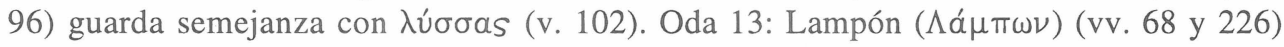

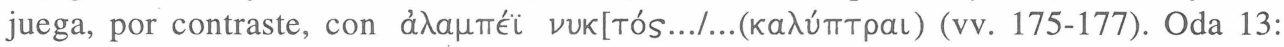

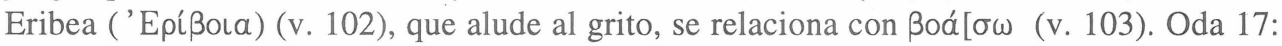

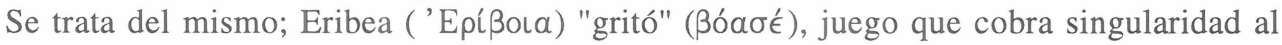
aparecer los términos en el mismo lugar (v. 14). Oda 17: Etra (Ä̈ $\rho \alpha$ ) (v. 59), madre de Teseo, alude al éter ('́s aíté $\rho a)$ (v. 73), indicando que no hay razones para la enemistad entre el cielo (Etra) -elemento paradójicamente de Minos- y el mar (Posidón). Y, quizás, el

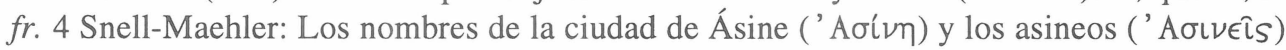
(v. 47) estarían relacionados con oívoual, porque los antiguos dríopes no volverían a causar daños en su nueva tierra?

4. Finalmente, la oda 19 (= ditirambo 5), titulada Ió y compuesta en honor de los atenienses, plantea el tema de la transformación de Ió, considerada tradicionalmente hija del río Ínaco y de la oceánide Melia, en novilla en su parte mítica (vv. 15-51), cuyos versos iniciales -que tienen el mismo tono interrogativo de la oda 15: "Musa, ¿quién fue el primero

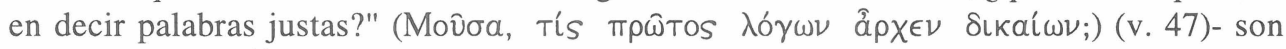
éstos (vv. 15-18) ${ }^{10}$ :

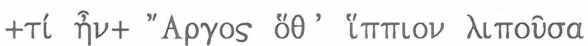

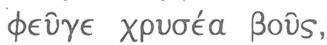

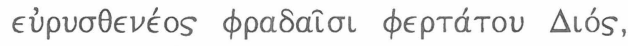

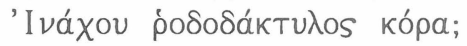

La versión que nos ha llegado a través de la tragedia, recogida por Esquilo (Supp. 291301), hace a Hera autora de la transformación de Ió, sacerdotisa y guardiana de su templo, para evitar que Zeus, enamorado, mantuviera relaciones con ella; pero no lo consiguió al convertirse el dios en toro. Sin embargo, la versión más extendida, recogida por Apolodoro (2.1.3), es la que da la noticia de que fue Zeus quien transformó a Ió en una novilla blanca para demostrarle a su esposa su inocencia.

\footnotetext{
${ }^{9}$ Cf. A. P. Burnett, op. cit. passim.

${ }^{10}$ Cf., e.g., R. C. Jebb, op. cit. 235-237, 398-405 y 492-496 y B. Snell-H. Maehler, op. cit. XLIX-L y 68-71.
} 
El problema es, pues, tratar de dilucidar en qué consistió ese cambio de Ió en novilla. a. En la versión más antigua, Ió sería transformada en una novilla (ßov̂s), que, normalmente, sería blanca ( $\lambda \in \cup \kappa \eta ́$ ) (Apolodoro [2.1.3] y Ovidio [Met. 1.652]). b. En el siglo V a.C. aparece como una doncella con cuernos de novilla, versión propia, sobre todo, de la tragedia, probablemente por la necesidad de que Ió pudiera hablar. c. En un período ya tardío y, según se documenta por testimonios arqueológicos, se volvería a la versión más antigua de la metamorfosis de Ió en novilla.

Por tanto, puede hablarse de la metamorfosis de Ió en novilla, añadiendo que ésta va a ser la versión más constante durante todas las épocas. La metamorfosis en doncella con cuernos de novilla puede ser más rara, sobre todo, por algo en lo que no se ha reparado: la metamorfosis de Ió en novilla lleva aparejada la metamorfosis de Zeus en toro para consumar la unión y, si bien es verdad que puede ser más habitual que una joven àarezca con atributos de animales, nunca lo hace Zeus, que se transforma en un animal completamente -son los casos del toro en el episodio de Europa o del cisne en el episodio de Leda-; cabría, pues, hablar mejor de la versión de la transformación de Ió en novilla.

Como antes quedó apuntado, la versión de la doncella con cuernos de novilla es propia de la tragedia con la que, entre otras cosas, se lograría que Ió hablara sin llamar demasiado la atención -desde luego, sería extraño ver en escena a una novilla capaz de hablar-. En Esquilo, que la incluyó en dos tragedias, las Suplicantes (463 a.C.) y Prometeo encadenado (último período del autor y, por tanto, antes del 456 a.C.), Ió es, fundamentalmente, una doncella con cuernos de novilla. La primera plantea dudas cuando el corifeo le contesta al rey

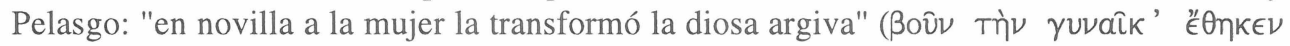

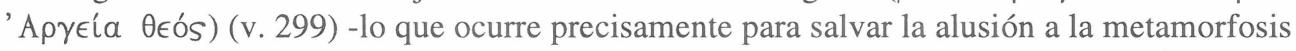
inmediata de Zeus-, pero es clara cuando el coro describe la impresión de los egipcios: "al contemplar a una res terrible, mezclada de ser humano, mitad de novilla y mitad, a su vez,

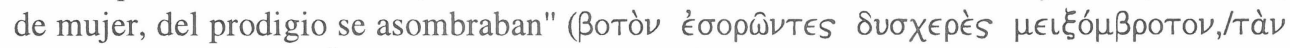

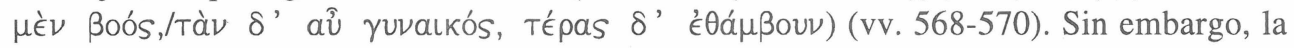
segunda se define sin ambages cuando Ió le pregunta a Prometeo, refiriéndose a sí misma:

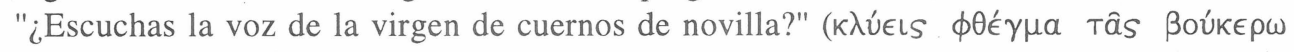
тар日évov;) (v. 588) y cuando Prometeo le responde: "¿Cómo no voy a escuchar a la

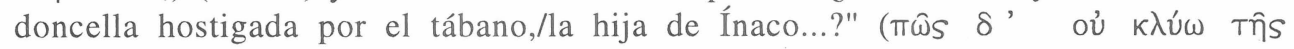

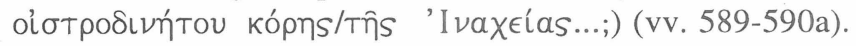

Resulta difícil ver qué tipo de transformación es la que aparece en Baquílides. Nuestro

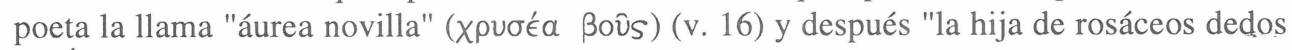

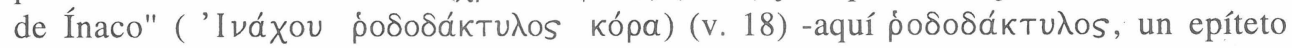
homérico típico de la Aurora, se aplica a Ió-. Parece, pues, que hay un primer momento de vacilación porque aparecen dos términos que hacen pensar en una doncella con cuernos de novilla: ßov̂s/kópa.

Los estudiosos que opinan que se trata de una doncella con cuernos de novilla ven en el

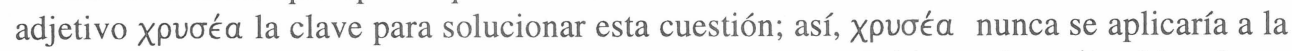
novilla en su totalidad, pues lo normal es considerar que sería blanca $(\lambda \in \cup \kappa \eta ́)$-al igual que 
aparece en Apolodoro-, sino que este epíteto haría referencia sólo a los cuernos: la unión de

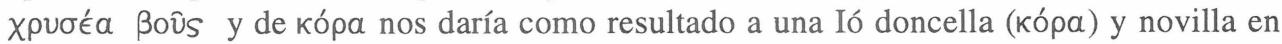

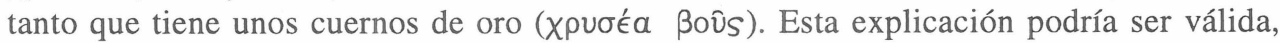
sobre todo, si se pone en relación con las tragedias de Esquilo.

Sin embargo, la otra explicación, quizás más sencilla, también parece coherente: Ió se ve

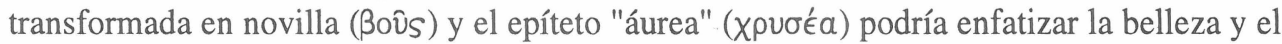
encanto de Ió como se hace con Afrodita, cuando se nos dice que Deyanira es "desconocedora

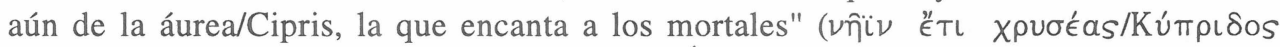
$\theta \in \lambda \xi\llcorner\mu \beta \rho o ́$ Tоv) (5.174-175) o como se hace con Ártemis, a la que se invoca de esta manera,

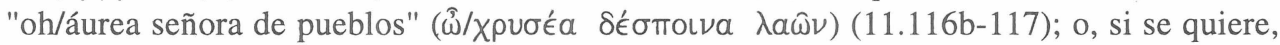
aun siendo novilla en su totalidad, destacaría lo áureo de sus cuernos y de ahí que en una sinécdoque se le aplicara el color de los cuernos a toda ella. También kópa, que significa "doncella", puede explicarse de dos maneras y ambas satisfactorias: por un lado, se hace referencia a una áurea novilla que, al fin y al cabo, no deja de ser en lo más hondo una doncella, una mujer, aunque esté metamorfoseada; por otro lado, lo más fácil quizás sea entender kópa como "hija", pues no por ser ahora una novilla deja de ser hija de Ínaco.

Podría verse en la oda un dato que permitiría pensar que la Ió de Baquílides es una novilla y no una doncella con cuernos de novilla, cuando se alude a ella como "la ternera de

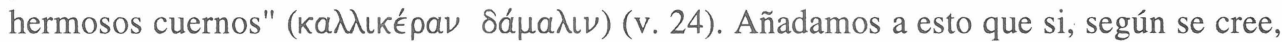
la versión de una Ió doncella con cuernos de novilla surge, en parte, por la necesidad de que intervenga en la tragedia y si se observa cómo en ningún momento de este poema, de fuerte estructura narrativa, se incluyen palabras en su boca, la opción de la metamorfosis total de la joven parece verosímil, aunque no podría descartarse sin ciertas reservas la otra.

5. La interpretación de una oda depende tanto del conocimiento preciso del material mítico que atesora como de la percepción nítida de la técnica poética que la configura. Se trata, pues, del reflejo claro de la unión del poeta y el público. Con este trabajo sólo se ha pretendido revisar algunos detalles de la obra de Baquílides, cuyo análisis permite valorar en mayor medida la riqueza de estos poemas corales, comparables, sin duda, a las creaciones de otros autores ${ }^{11}$.

${ }^{11}$ Cf. R. C. Jebb, op. cit. 27-55. 